\title{
Xanthogranulomatous pyelonephritis due to calculi in a 5-year-old girl
}

\author{
Esra Nagehan Akyol Önder ${ }^{1 \oplus}$, Mine Özkol $^{2 \odot}$, Nalan Neşe $^{3 \odot}$, Can Taneli $^{4 \oplus}$, \\ İpek Özünan ${ }^{1 \odot}$
}

Departments of ${ }^{1}$ Pediatric Nephrology, ${ }^{2}$ Radiology, ${ }^{3}$ Pathology and ${ }^{4}$ Pediatric Surgery, Celal Bayar University, Faculty of Medicine Manisa, Turkey.

\begin{abstract}
Background. Xanthogranulomatous pyelonephritis is characterized by the inflammatory destruction of the renal parenchyma and intensive renal fibrosis. It is named because of its pathological appearance; that of its granulomatous inflammatory process with lipid-laden macrophages, which appear yellow, hence 'xantho' which is Greek for yellow. Xanthogranulomatous pyelonephritis is predominantly a disease of adults. In children it is diagnosed sporadically and is extremely rare in infants. The age of onset varies (21 days to 16 years), although $60-75 \%$ of cases have been diagnosed before 5 years of age. Recurrent urinary tract infections, obstructive nephropathy caused by renal calculus, malnutrition, abnormal lipid metabolism, altered immunologic response, lymphatic blockage, congenital urinary anomalies have been implicated in the etiology of xanthogranulomatous pyelonephritis in children.
\end{abstract}

Case. We report an unusual case of xanthogranulomatous pyelonephritis in a 5-year-old girl and discuss its clinical features, histopathological findings and treatment. In this article, we also emphasized the importance of diagnostic imaging in urinary tract infections which enabled us not to miss the underlying kidney stone disease.

Conclusion. Nephrolithiasis may lead to very serious conditions such as xanthogranulomatous pyelonephritis. This condition can be easily diagnosed by ultrasound, but if not detected, it can lead to complete loss of renal function as in the case.

Key words: Xantogranulomatous pyelonephritis, nephrolithiasis, kidney stones.

Xanthogranulomatous pyelonephritis $(\mathrm{XPN})$ is an unusual and severely chronic inflammatory disease of the kidney, characterized by infiltration of the renal parenchyma with lipid laden macrophages. ${ }^{1}$ It is rarely seen in childhood and the mechanism of XPN is poorly understood. ${ }^{2}$ Recurrent urinary tract infections, obstructive nephropathy caused by renal calculus, malnutrition, abnormal lipid metabolism and altered immunologic response

$\bowtie \quad$ Esra Nagehan Akyol Önder esra.nagehan.7@hotmail.com

Received 17th September 2019, revised 14th November 2019, 29th December 2019, accepted 18th February 2020.

This case was presented at the 10th Turkish National Congress of Pediatric Nephrology, 1-4 May 2019, Bodrum, Turkey. reported to predispose an individual to this rare renal parenchymal infection often mimicking neoplastic renal disorder. ${ }^{3}$

\section{Case Report}

A five-year-old girl with complaints of flank pain, concomitant hyperpyrexia, loss of appetite for a month was referred to our clinic for further evaluation. She was treated with several courses of antibiotics for about a month in different centers. However, no diagnostic imaging was performed. Her history revealed that her development was uneventful. There was a positive family history of nephrolithiasis.

Her physical examination findings were as follows: weight, $15 \mathrm{~kg}$ ( $<3$ percentiles); height, 
$110 \mathrm{~cm}$ (50-75 percentiles); heartbeat, 80/ min; respiratory rate, $22 / \mathrm{min}$; blood pressure, $90 / 50 \mathrm{mmHg}(<90 \mathrm{p})$. A mass on the left side extending beyond the midline into the pelvis with dimensions of $8 \times 10 \mathrm{~cm}$ was palpated. Costovertebral angle tenderness was not detected. Physical examination of other systems was unremarkable.

Her laboratory findings were as follows: white blood cell count 28420/mm3; platelets 752000/ $\mathrm{mm} 3$. Peripheral blood smear findings revealed polymorphonuclear leucocytes $(66 \%)$, toxic granulation, and hypochromia (18\%). Acute phase reactants were also elevated: erythrocyte sedimentation rate: $120 \mathrm{~mm} / \mathrm{h},(0-15 \mathrm{~mm} / \mathrm{h})$, C-reactive protein: $15 \mathrm{mg} / \mathrm{L} \quad(0-0,5 \mathrm{mg} / \mathrm{dl})$ and procalcitonin: $0,34 \mathrm{ng} / \mathrm{ml},(0-0,05 \mathrm{ng} / \mathrm{ml})$. Biochemical values were within normal limits. Urine analysis showed: urine density, 1026; $\mathrm{pH}$, 6,5; leukocytosis and intermittent haematuria. Urine culture was positive for Pseudomonas spp. and it was susceptible to ceftazidime.

On abdominal ultrasonography (USG), the left kidney was enlarged diffusely (111x61 $\mathrm{mm})$. Five to six stones within the left pelvis renalis measuring $9 \mathrm{~mm}$, and a few millimetric calculi within of the calyceal groups were detected. The pelvic-calyceal lumens appeared dilated and full of pus. There was inflammatory hyperechogenicity in perirenal adipose tissue. The right kidney was normal in size and echogenicity. Abdominal computed tomography (CT) was consistent with left renal multiple pelviceal stones, diffuse enlargement, foci of calcifications and hydro-pyonephrosis (Fig.1A). In a technetium 99m-dimercaptosuccinic acid renal scan a nonfunctional left kidney was revealed (Fig.1B).

Based on the clinical examination and imaging, the differential diagnosis included renal tuberculosis, Wilms tumor and XPN. Three early-morning urine samples sent for acidfast stain were negative. Tuberculin skin test was negative. The patient had no concomitant clinical or radiographic findings suggestive of pulmonary involvement, thus we ruled out renal tuberculosis. We examine the patient for Wilms tumor associated anomalies, such as aniridia, hemihypertrophy, and genitourinary anomalies but we didn't find any signs related with these anomalies. Because of the suspected renal tumor, Doppler USG was performed for detecting tumor infiltration in the renal vein or inferior vena cava but no evidence was found. Additionally, chest radiograph and abdominal CT was negative for metastases. Although it is not easy to diagnose XPN preoperatively, based on the patient's typical clinical presentation of drug resistant urinary tract infection due to calculi and the combination of a nonfunctioning enlarged kidney, multiple central stones within the contracted renal pelvis, expansion of the calices, and inflammatory changes in the perinephric fat on the imaging features, a clinical diagnosis of XPN was made and a left radical nephrectomy was performed. No complication was observed during the operation and postoperative period. In the macroscopic examination of the specimen, left nephrectomy material was noted as having $11,5 \times 7 \times 6,5 \mathrm{~cm}$ dimensions and weighing $225 \mathrm{~g}$. The outer layer of the specimen was multilobulated. There was a remarkable loss of renal parenchyma. All of the calyceal lumens were dilated and full of yellow-colored pus and multiple stones (Fig.1C). The sample tissues were fixed in 10\% formalin and embedded in paraffin. Sections cut at $4 \mathrm{~mm}$ were stained with hematoxylin-eosin. Histopathological examination showed an acute and chronic inflammatory cell infiltrated with giant cells and lipid-laden macrophages (foam cells) on cortical areas (Fig. 1D). After surgery, she was feeling well and gained weight. Her acute phase reactants decreased to normal. She has been followed up at threemonth intervals for nearly 1 year after surgery without complications. Informed consent was received from the family.

\section{Discussion}

Xanthogranulomatous pyelonephritis is an uncommon chronic obstructive pyelonephritis characterized by infiltration and fibrosis of 

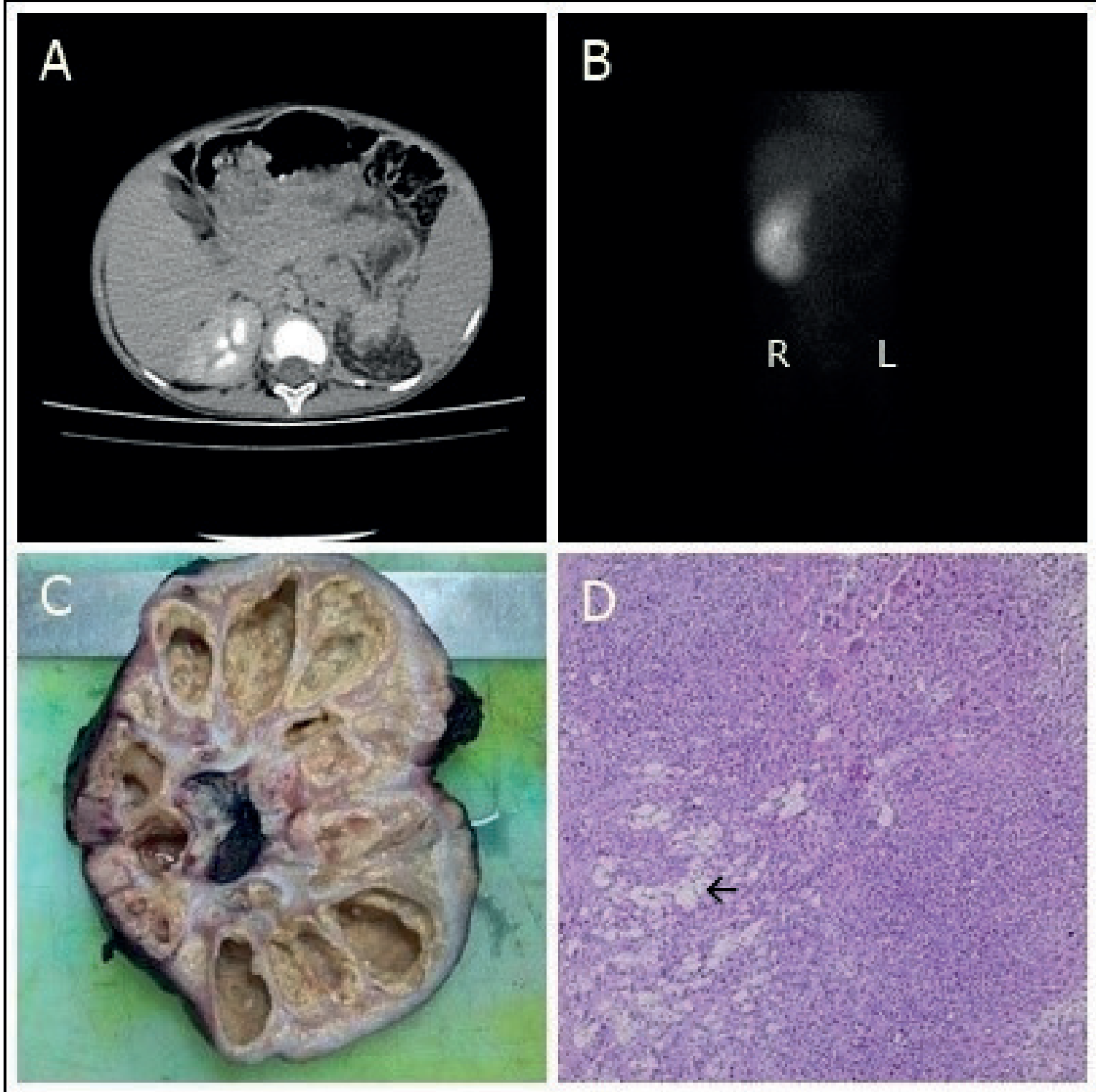

Fig. 1. (A) Computed tomography (CT) consistent with left renal multiple pelviceal stones, diffuse enlargement, foci of calcifications and hydro-pyonephrosis. (B) DMSA scan shows non-functional left kidney. (C) Macroscopic features of XPN: dilated calyces filled with pus and yellow tinge of fat laden macrophage layer is seen. (D) Histological features of XPN showing fibrosis, inflammatory infiltrate and the black arrow points fat laden macrophages.

the renal parenchyma composed of lipid laden macrophages, as well as a variety of inflammatory elements including, neutrophils ,lymphocytes, plasma cells, cholesterol clefts and multinucleated giant cells. ${ }^{1,2,4}$ Recurrent urinary tract infections with E.coli, Proteus mirabilis, and rarely Pseudomonas species (54\%), obstructive nephropathy caused by renal calculus (68\%), malnutrition, abnormal lipid metabolism, altered immunologic response (5\%), lymphatic blockage, congenital urinary anomalies (5\%) have been reported to predispose an individual to this rare renal parenchymal infection often mimicking neoplastic renal disorder. ${ }^{1,3}$ XPN has been termed "the great imitator" because the differential diagnosis 
includes a large group of disease such as Wilms tumor, renal cell carcinoma, renal abscess, infected renal cystic disease, tuberculosis, malakoplakia, and transitional renal cell carcinoma. The preoperative diagnostic rates are higher in the pediatric population. ${ }^{5}$

Schlagenhaufer first described features of XPN in $1916 .{ }^{1}$ Quinn et $a .^{6}$ reported the presence of multiple calculi in 26 out of 31 patients involving the left kidney. In the same study, affected kidneys of 23 cases were detected as being nonfunctional or inadequately functional $(<10 \%) .{ }^{6}$ Our patient had a non-functional left kidney with multiple calculi and her urine culture was positive for Pseudomonas spp.

Bingol-Koloğlu et al. ${ }^{7}$ reported that $\mathrm{XPN}$ is predominantly a disease of adults however it may occur in all age groups with two peaks, one before the age of 10 and the other in the fourth to fifth decade. Only a third of all cases were seen in children and they found a male predominance $(85 \%)$. However, there are some reports revealing that the diffuse form is seen equally in boys and girls while focal XPN is more common in girls. ${ }^{8-10}$

Histologically the disease is characterized by lipid laden foamy macrophages and acute and chronic renal inflammatory cells.

Treatment is always surgical excision of the damaged tissue (total or partial nephrectomy) depending on the extent of involvement and the prognosis after the surgery is excellent.

In conclusion, $\mathrm{XPN}$ is a rare condition in children, the symptoms are usually mild. It is a histopathological entity and preoperative diagnosis is challenging; thus it is difficult to diagnose. It should be kept in mind that fever and flank tenderness are the most common symptoms. The mainstay imaging technique is CT in clinically suspected patients. The definitive diagnosis of XPN is pathological. Nephrectomy is the curative treatment. In this article, we also emphasized the importance of diagnostic imaging in recurrent urinary tract infections. It is necessary to diagnose underlying kidney stone disease. Nephrolithiasis may lead to very serious conditions such as XPN. They can be easily diagnosed by ultrasound, but if not detected, this can lead to XPN and complete loss of renal function as in this case.

\section{Acknowledgements}

We thank the parents of the patient described for allowing us to share her details.

\section{REFERENCES}

1. Sangüesa Nebot C, Pico Aliaga S, Serrano Durba A, Roca MJ. Xantogranulomatous pyeloneprhritis in children. Insights Imaging 2018; 9: 643-651.

2. Addison B, Zargar H, Lilic N, Merrilees D, Rice M. Analysis of 35 cases of xanthogranulomatous pyelonephritis. ANZ J Surg 2015; 85: 150-153.

3. Samuel M, Duffy $P$, Capps S, Mouriquand P, Williams D, Ransley D. Xanthogranulomatous pyelonephritis in childhood. J Pediatr Surg 2001; 36: 598-601.

4. Li L, Parwani AV. Xanthogranulomatous pyelonephritis. Arch Pathol Lab Med 2011; 135: 671674.

5. Zugor V, Schott GE, Labanaris AP. Xanthogranulomatous pyelonephritis in childhood: a critical analysis of 10 cases and of the literature. Urology 2007: 70: 157-160.

6. Quinn FMJ, Dick AC, Corbally MT, McDermott MB, Guiney EJ. Xanthogranulomatous pyelonephritis in childhood. Arch Dis Child 1999; 81: 483-486.

7. Bingol-Kologlu M, Ciftci AO, Senocak ME, Tanyel FC, Karnak I, Buyukpamukcu N. Xanthogranulomatous pyelonephritis in children: diagnostic and therapeutic aspects. Eur J Pediatr Surg 2002; 12: 4248 .

8. Braun G, Moussali L, Balanzar JL. Xanthogranulomatous pyelonephritis in children. J Urol 1985; 133: 236-239.

9. Clapton WK, Boucaut HAP, Dewan PA, Bourne AJ, Byard RW. Clinicopathological features of xanthogranulomatous pyelonephritis in infancy. Pathology 1993; 25: 110-113.

10. Hammadeh MY, Nicholls G, Calder CJ, Buick RG, Gornall P, Corkery JJ. Xanthogranulomatous pyelonephritis in childhood: pre-operative diagnosis is possible. Br J Urol 1994; 73: 83-86. 